\title{
Non-tuberculous mycobacterial keratitis: a study of 22 cases
}

\author{
Samuel C M Huang, H Kaz Soong, Jen-Shiang Chang, Yu-Sung Liang
}

\begin{abstract}
Aim-To investigate causes and clinical findings of non-tuberculous mycobacterial keratitis, and to study its response to topical antibiotic therapy and surgical extirpative keratectomy.

Method-A single centre, retrospective review of 22 patients with non-tuberculous mycobacterial keratitis seen in a 3 year period. Laboratory diagnoses were established with Ziehl-Nielsen acid fast staining and Löwenstein-Jensen cultures.

Results-In 20 patients $(91 \%)$, there was an antecedent history of foreign body eye trauma (18 patients) or elective surgery (two patients). There were 19 cases of Mycobacterium chelonei, and three of $M$ fortuitum. Clinical signs included epithelial defects, satellite or ring stromal infiltrates, crystalline keratopathy, and hypopyon. For topical antibiotic therapy, 20 patients received amikacin, while one patient received rifampin and another received ciprofloxacin, each in accordance with the results of the in vitro drug sensitivities. An extirpative keratectomy was performed in 15 cases; four of these cases additionally required a temporary conjunctival flap in order to finally eradicate the infection. At the end of the follow up period (median 18 months; range 3 months to 3 years) all eyes were stable and free of infection, with $19(86 \%)$ having final visual acuities of $20 / 200$ or better. Conclusion-Early clinical recognition and prompt laboratory diagnosis, together with aggressive topical antibiotic therapy and early keratectomy, may shorten morbidity and improve the clinical outcome of non-tuberculous mycobacterial keratitis.
\end{abstract}

(Br F Ophthalmol 1996;80:962-968)

Non-tuberculous or atypical mycobacteria are widespread in the environment ${ }^{1}$ and were once thought to be non-pathogenic. Nontuberculous mycobacteria are now known to cause a variety of human diseases, including infections of the cornea. In 1965, Turner and Stinson $^{2}$ described the first case of nontuberculous mycobacterial keratitis in a corneal ulcer that developed 4 months after removal of a superficial foreign body; Mycobacterium fortuitum was identified as the aetiological agent of the ulcer. The first case of corneal infection with $M$ chelone $i$ was reported by Gangadharam and coworkers ${ }^{3}$ in 1978. Several subsequent reports of $M$ fortuitum and $M$ chelonei keratitis have been recorded in the literature. ${ }^{4-14}$ Corneal infections with $M$ flavescens, $M$ avium-intracellulare, $M$ gordonae, and $M$ marinum have also been reported, but appear to be quite infrequent in comparison with $M$ fortuitum and $M$ chelonei. ${ }^{3}{ }^{14-17}$ Most reported corneal infections by non-tuberculous mycobacteria are associated with trauma or surgery.

Topical fortified amikacin $(14-100 \mathrm{mg} / \mathrm{ml})$ is the drug of choice in the treatment of most non-tuberculous mycobacterial corneal infections. ${ }^{418-21}$ The non-tuberculous mycobacteria also show varying degrees of susceptibility to other drugs, including the fluoroquinolones, aminoglycosides, tetracycline family, erythromycin, cephalosporins, sulphonamides, neomycin, clarithromycin, and vancomycin. ${ }^{91820-22}$

We report herein 22 culture proved cases of infectious keratitis caused by non-tuberculous mycobacteria. The purpose of this study was to investigate the causes and clinical findings in non-tuberculous mycobacterial corneal infection, and to study its response to topical therapy and surgical keratectomy.

\section{Materials and methods}

Between January 1991 and June 1994, we investigated 22 consecutive cases of nontuberculous mycobacterial keratitis. The study included reviews of the patient charts at the Chang Gung Memorial Hospital and at the referring ophthalmologists' offices, interviews with the patients and the referring ophthalmologists, microbiological cultures of the infected corneas, drug sensitivity tests for commonly used antibiotics and for antituberculous agents, and pathological examination of extirpative lamellar keratectomy specimens.

\section{DIAGNOSTIC CRITERIA}

Positive non-tuberculous mycobacterial cultures on Löwenstein-Jensen agar medium constituted a definitive diagnosis of nontuberculous mycobacterial keratitis. Although these organisms may often grow on standard media, Löwenstein-Jensen medium provides much more optimal and specific growth conditions for mycobacteria. Whether or not the smears for acid fast organisms are positive, the material should be cultured for several reasons: (1) cultures can detect lower quantities of the organism, and (2) tests for drug sensitivities will be useful in directing medical treatment. All 22 patients had positive cultures for non-tuberculous mycobacteria. Despite positive Löwenstein-Jensen cultures, only 10 of these specimens showed acid fast bacteria with the Ziehl-Nielsen stain. An antecedent history 
of trauma, especially superficial corneal foreign body injuries, greatly increased the index of suspicion of infection with this microorganism. Negative routine cultures for bacteria, herpes simplex virus, fungus, and Acanthamoeba, together with lack of clinical response to routine antibiotic (and occasionally corticosteroid) therapy, usually helped confirm our suspicions. Routine culture methods included the use of the following media: blood agar, chocolate agar, thioglycollate broth, and Sabouraud dextrose agar. Whenever necessary, herpes simplex cultures were transported in Hanks's balanced salt medium and inoculated on rabbit kidney cells, and Acanthamoeba was cultured on non-nutritive agar with Escherichia coli confluent overlay. Direct smears for Gram and Ziehl-Nielsen stains were prepared in each case. In two cases, a 'cracked windshield' corneal infiltrate pattern was virtually diagnostic in itself. As we encountered more cases of non-tuberculous mycobacterial corneal infections in our practice, not only did our diagnostic acumen become honed, but we also began to realise that this organism was not really as rare as we had once assumed.

\section{LABORATORY DIAGNOSTIC PROCEDURE}

Corneal specimens for culture were obtained by superficial scraping of stromal infiltrates with a Kimura spatula. These were inoculated onto Löwenstein-Jensen medium and were maintained for over 4 weeks in some cases.

In the cases which required extirpative corneal surgery, part of the keratectomy specimen was placed in formalin for histopathological examination with haematoxylin and eosin and Ziehl-Nielsen acid fast stains and part was sent to the microbiological laboratory for Löwenstein-Jensen cultures and for antibiotic sensitivity assays. The broth microdilution method was used for the antibiotic susceptibility testing.

\section{TREATMENT}

Non-tuberculous mycobacteria are generally resistant to conventional antibiotics, but they do respond favourably to amikacin. Initial conventional antibiotics used routinely by us consisted of fortified topical gentamicin and cefazolin. Varying degrees of in vitro susceptibility have been reported to a broad range of other agents including fluoroquinolones, aminoglycosides, tetracycline derivatives, erythromycin, vancomycin, cephalosporins, and sulphonamides.

Once the diagnosis was established by culture, our mainstay of treatment was topical amikacin $50 \mathrm{mg} / \mathrm{ml}$ hourly around the clock (20 patients). In one patient (case 5), only topical rifampin $(30 \mathrm{mg} / \mathrm{ml})$ was used, as drug sensitivity tests indicated a susceptibility to rifampin only. In another patient (case 11), only topical ciprofloxacin was used (once again in accordance with the drug sensitivity assay). Once the diagnosis of non-tuberculous mycobacterial keratitis was established by laboratory methods, antimicrobial therapy was altered appropriately.
Extirpative keratectomy was required in severe or recalcitrant cases in order to reduce or eliminate the numbers of infectious organisms in the cornea. This also ostensibly enhanced the antimicrobial drug penetration and improved patient comfort. Furthermore, the surgical excision provided an excellent biopsy source. Keratectomies were performed under retrobulbar anaesthesia with the aid of an operating microscope. A slightly oversized, round zone marker was used to delineate the infected area to be excised. A No 64 or No 69 Beaver blade was used to make the initial vertical incision up to about two thirds of the corneal depth. Either a No 57 or No 66 Beaver blade was then used for the intralamellar dissection of the infected and/or necrotic stroma.

If the infection failed to respond to extirpative surgery and intensive antimicrobial therapy, a Gundersen conjunctival flap was performed (four patients). In all cases, the conjunctival flaps were successful in controlling the infection and stabilising the eye. The conjunctival flaps were taken down after 6 months, leaving corneas with varying degrees of scarring. One case eventually underwent a successful penetrating keratoplasty following takedown of the conjunctival flap (case 17).

\section{Results}

DEMOGRAPHICS AND CLINICAL HISTORY

The patients ranged in age from 20 to 66 years (median 38.5 years). The median follow up period was 18 months (range 3 months to 3.5 years). The majority ( $82 \%)$ of the patients were male. In 20 of the 22 patients ( $91 \%$ ), there was an antecedent traumatic or surgical history. This subgroup of 20 patients was more specifically divided into 18 who had trauma and two who had surgery. All 18 trauma cases involved superficial corneal foreign body injuries, the majority with metallic agents $(61 \%)$ and the remainder with mineral or vegetable matter. The two postsurgical corneal infections occurred within 1 year of elective eye surgeryone was associated with pterygium excision (bare sclera technique) (case 17) and the other was associated with a failed corneal graft (originally done for contact lens related Pseudomonas keratitis) (case 4).

Eighteen of 22 patients $(82 \%)$ were male, paralleling the predominance of traumatic aetiologies in most cases. Twelve cases were associated with foreign body injuries incurred at work, thus illustrating the importance of this problem among the blue collar workforce and the need for enforcing adequate eye protection in the workplace. In the non-traumatic cases, one had chronic ocular surface disease associated with cicatricial pemphigoid (case 2) and the other had neurotrophic keratopathy after excision of a cerebropontine angle tumour (case 5).

Typically, the clinical symptoms and findings of corneal infection did not appear immediately after corneal injury. The time interval between onset of trauma and appearance of the corneal infection ranged from 3 days to 3 weeks. Time of diagnosis from the onset of 
keratitis ranged from 1 week to 2 months. Generally, as we gained more experience in the diagnosis and management of non-tuberculous mycobacterial keratitis, this latter time interval became shorter.

\section{MICROBIOLOGY}

Nineteen of 22 cases were culture positive for $M$ chelonei, while three were culture positive for $M$ fortuitum. Typical histopathological findings are shown in Figure 1.

In vitro minimal inhibitory concentrations (MIC) for amikacin in the 20 susceptible cases ranged from 9 to $15 \mu \mathrm{g} / \mathrm{ml}$. In cases 5 and 11 , the MIC indicated resistance to amikacin, but good sensitivity to either rifampin (case 5) or ciprofloxacin (case 11). Antibiotic resistance was indicated by the following criteria: (1) amikacin $>64 \mu \mathrm{g} / \mathrm{ml}$, (2) rifampin $1 \mu \mathrm{g} / \mathrm{ml}$, and (3) ciprofloxacin $0.8 \mu \mathrm{g} / \mathrm{ml}$.

In total, keratectomies were performed in 15 out of 22 patients (68\%). This included seven patients in whom the organisms showed good sensitivity in vitro to the antibiotics employed. In these patients, the infection was so severe and deep that drug therapy alone was not sufficient to eradicate the disease.

In some cases, the aetiology of keratitis was initially mistaken for fungus, herpes simplex, or Acanthamoeba, as the clinical findings were often masquerades for these agents. Poor clinical responses to therapy, together with negative fungal, viral, and parasitic laboratory studies, were helpful (albeit belatedly) in establishing the final correct diagnosis. No cases of mixed infections involving non-tuberculous mycobacteria and other organisms were encountered.

Surprisingly, only $11(50 \%)$ of the scrapings showed positive Ziehl-Nielsen acid fast staining. The Löwenstein-Jensen cultures, on the other hand, were positive for all 22 patients. This may be due to: (1) low numbers of organisms not adequately detected by the smear, but detected after 'amplification' provided by cultivation; (2) tendency by the clinician to prioritise the specimen for culture, rather than for simple smearing, when the available corneal inoculum is sparse; and/or (3) acid wash being too potent in the Ziehl-Nielsen method, leading to overdecolorisation of the organisms (see Discussion for alternative staining method, the Fite-Ferraco stain). This experience re-emphasised the importance of obtaining culture identification, rather than depending solely on the acid fastness criterion. Definitive mycobacteria were not seen on retrospective examination of the Gram stain specimens.

\section{CLINICAL FINDINGS}

In all cases, except for the one with underlying neurotrophic keratopathy, the patients complained of moderate pain and photophobia. Excruciating pain, characteristic of Acanthamoeba keratitis, was not encountered. Varying degrees of blurring were noted by the patients.

Clinical signs are listed in Table 1. All patients showed persistent corneal epithelial defects and underlying inflammatory stromal infiltrates at various depths. Eight patients manifested a main stromal infiltrate surrounded either by discrete satellite infiltrates or fluffy extensions of the parent infiltrate. Six patients showed either a complete or partial ring infiltrate. Three patients manifested crystalline keratopathy, including two who had a cracked windshield stromal lesion (Fig 2), considered by some to be diagnostic of nontuberculous mycobacterial keratitis. ${ }^{121723}$ Vary-

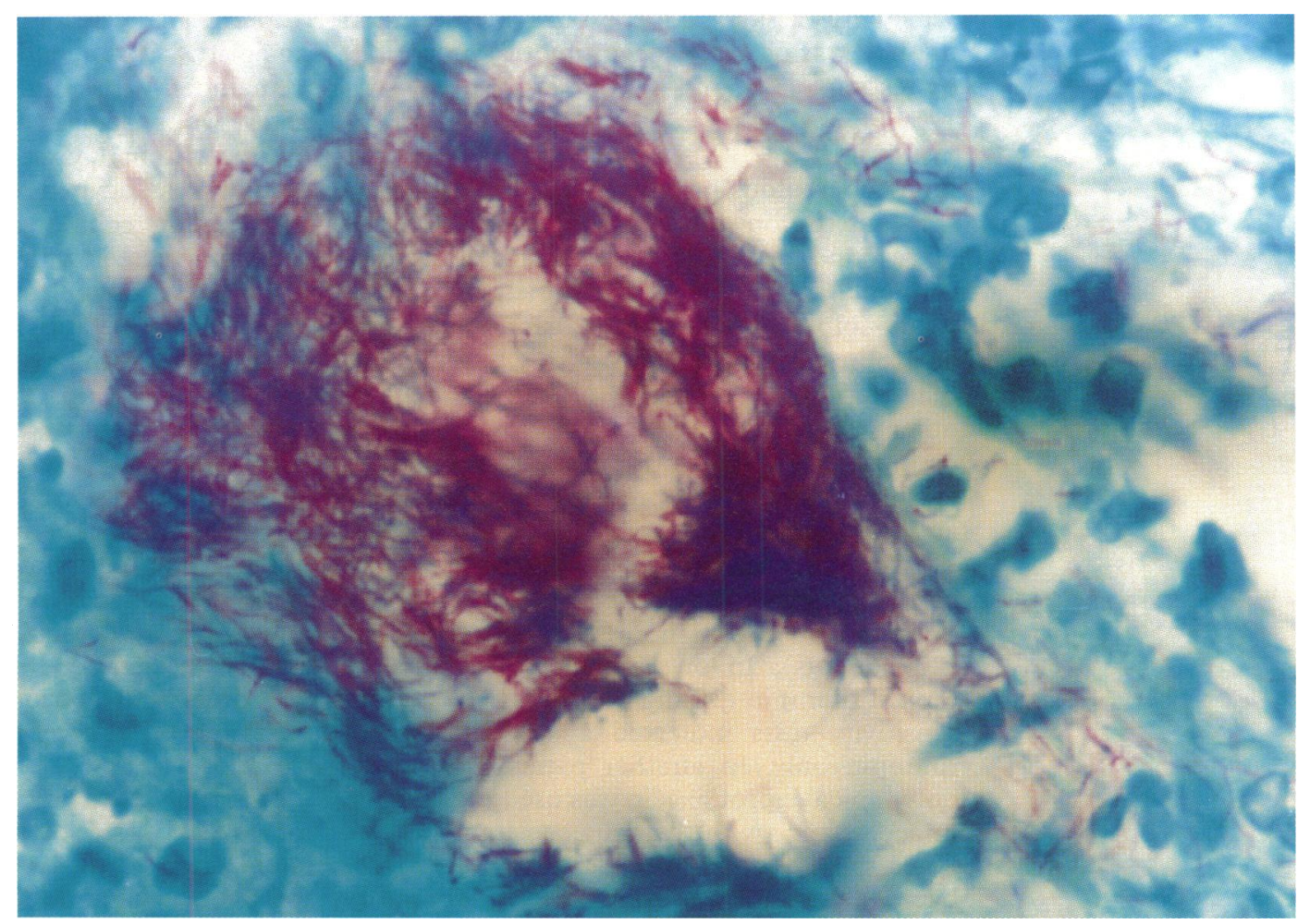

Figure 1 Histopathological appearance of a colony of $M$ chelonei in an anterior lamellar keratectomy specimen, showing acid fast mycobacteria. (Ziehl-Nielsen stain; original magnification $\times 1584)$. 


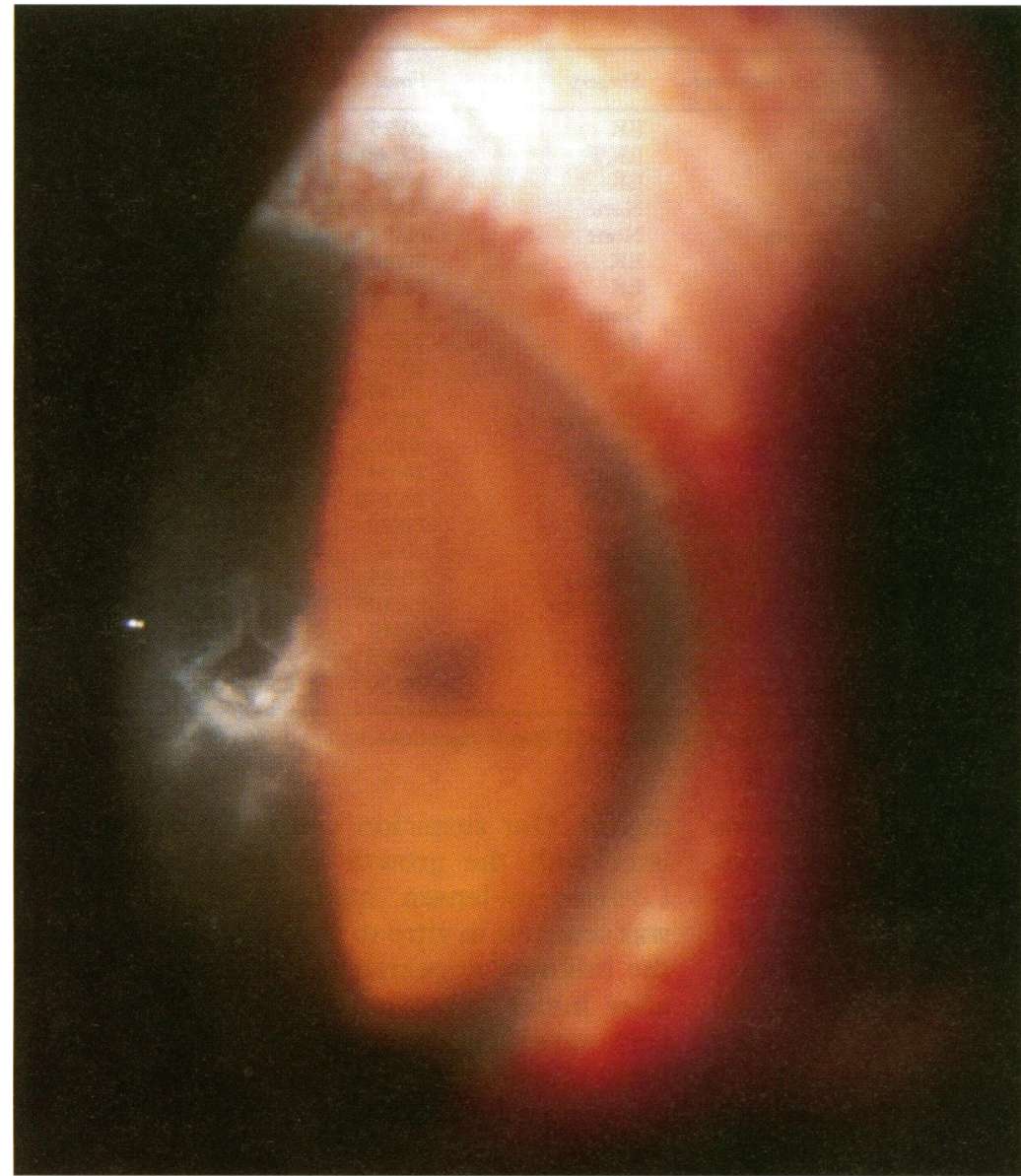

Figure 2 Slit-lamp biomicroscopic appearance of cracked windshield corneal infiltrate pattern (case 20). large epithelial defect centrally with a large, underlying disciform, intrastromal white cell infiltrate. A $10 \%$ layered hypopyon was present in the anterior chamber. The lens appeared clear, but the fundus could not be adequately visualised because of the corneal opacity. Intraocular pressures by applanation tonometry were normal. The corneal sensation was absent in the right eye.

Bacterial and fungal studies were obtained on corneal scrapings from the right eye, and the patient was started on hourly doses of topical cefazolin $50 \mathrm{mg} / \mathrm{ml}$. Routine Gram stains and cultures were negative, and Ziehl-Nielsen staining was negative for acid fast organisms. The corneal ulcer responded poorly to therapy. The Löwenstein-Jensen culture grew $M$ chelonei 3 weeks after inoculation. The organism was sensitive in vitro only to rifampin. The cefazolin eyedrops were discontinued and the patient was started on hourly doses of topical rifampin $30 \mathrm{mg} / \mathrm{ml}$. The corneal infiltrates responded gradually to this regimen and medication was tapered over a 1 month period. In the setting of neurotrophic and exposure keratopathy, the epithelial defect persisted and the hypopyon never completely disappeared. One month after cessation of the eyedrops, a new stromal infiltrate appeared in the central cornea in the right eye. Reinstitution of hourly topical rifampin resulted in complete resolution of the infection, leaving a dense central corneal stromal scar. Six months later, the patient underwent penetrating keratoplasty in the right eye, but owing to a persistent epithelial defect, the graft eventually failed.

ing degrees of anterior chamber inflammatory reactions were seen in eight patients, of which five had hypopyon. No cases of infectious endophthalmitis or scleritis were seen.

\section{CLINICAL COURSE}

The final best corrected visual acuity after eradication of the infection and after all the associated surgeries ranged from hand movements to 20/20 (Table 2). In 19 patients $(86 \%)$, the best corrected visual acuity was $20 / 200$ or better. The remaining three patients with poorer visual acuities have not undergone vision restoring penetrating keratoplasties for two reasons: (1) their contralateral eyes were good enough to allow adequate performance of daily visual tasks, and (2) good donor corneal tissue is relatively difficult to obtain in Taiwan.

REPORTS OF CASES

Case 5

A 47-year-old woman underwent craniotomy on 8 October 1993 for excision of a benign cerebellopontine angle tumour. Subsequently, she developed right hemifacial palsy with lagophthalmos and severe right neurotrophic keratopathy. Two weeks later, she was sent to us for evaluation and treatment of a corneal ulcer in her right eye. The visual acuities were hand movement in the right eye and 20/20 in the left. Significant lagophthalmos and corneal exposure were noted in the right eye. The conjunctiva in the right eye was very injected. Slitlamp examination of the right cornea showed a

\section{Case 7}

A 62-year-old mechanic sustained a superficial metallic foreign body injury to his left eye while working. The foreign body was removed by the factory medical staff; however, the patient continued to feel intermittent foreign body sensation in this eye for about 2 weeks. When the pain persisted, he was referred to our clinic for further evaluation. In the right eye, the visual acuity was $20 / 400$ and the conjunctiva was severely inflamed. A $3 \times 3 \mathrm{~mm}$ peripheral corneal epithelial defect, with an underlying, ringshaped, anterior stromal infiltrate, was present near the limbus in the 1 o'clock meridian. The ring infiltrate was surrounded by several small satellite infiltrates. A provisional diagnosis of herpetic keratitis was made and the patient was started on acyclovir ophthalmic ointment four

Table 1 Clinical signs of non-tuberculous mycobacterial keratitis

\begin{tabular}{ll}
\hline Clinical sign & $\begin{array}{l}\text { No of } \\
\text { cases }\end{array}$ \\
\hline Corneal epithelial defect & 22 \\
Corneal stromal infiltrates & 22 \\
Disciform infiltrates & 8 \\
Main infiltrate surrounded by satellites & 8 \\
Partial or full ring infiltrates & 6 \\
Crystalline keratopathy (including cracked & 3 \\
windshield infiltration) & 8 \\
Iritis & 5 \\
Hypopyon & 0 \\
Infectious endophthalmitis & 0 \\
Scleritis & \\
\hline
\end{tabular}


Table 2 Case summaries of non-tuberculous mycobacterial keratitis

\begin{tabular}{|c|c|c|c|c|c|c|c|}
\hline No & Age/sex & Nature of corneal insult & Organism & Topical treatment & Surgery & Final VA & Acid fastness * \\
\hline 1 & $39 / M$ & Metallic FB & $M$ chelonei & Amik & EK & $20 / 25$ & + \\
\hline 2 & $61 / M$ & Pemphigoid & $M$ chelonei & Amik & $\mathrm{EK} / \mathrm{F}$ & $\mathrm{CF} 60 \mathrm{~cm}$ & - \\
\hline 3 & $47 / M$ & Metallic FB & M chelonei & Amik & EK & $20 / 50$ & + \\
\hline 4 & $62 / M$ & Failed graft & $M$ chelonei & Amik & None & $\mathrm{HM} 60 \mathrm{~cm}$ & - \\
\hline 5 & $47 / \mathrm{F}$ & Neurotrophic/exposure & $M$ chelonei & Rifampin & None & HM $60 \mathrm{~cm}$ & - \\
\hline 6 & $20 / M$ & Unknown FB & M chelonei & Amik & EK & $20 / 40$ & + \\
\hline 7 & $62 / M$ & Metallic FB & $M$ fortuitum & Amik & EK & $20 / 100$ & - \\
\hline 8 & $49 / \mathrm{F}$ & Unknown FB & $M$ chelonei & Amik & None & $20 / 40$ & - \\
\hline 9 & $38 / \mathrm{M}$ & Unknown FB & M chelonei & Amik & EK/F & $20 / 200$ & - \\
\hline 10 & $31 / \mathrm{M}$ & Metallic FB & $M$ chelonei & Amik & EK & $20 / 20$ & - \\
\hline 11 & $36 / \mathrm{M}$ & Clay FB & $M$ fortuitum & Cipro & EK & $20 / 40$ & + \\
\hline 12 & $25 / M$ & Metallic FB & $M$ chelonei & Amik & EK twice & $20 / 30$ & + \\
\hline 13 & $33 / \mathrm{M}$ & Unknown FB & $M$ fortuitum & Amik & None & $20 / 200$ & + \\
\hline 14 & $45 / M$ & Metallic FB & M chelonei & Amik & None & $20 / 50$ & - \\
\hline 15 & $66 / M$ & Wood FB & $M$ chelonei & Amik & EK/F & $20 / 200$ & + \\
\hline 16 & $30 / \mathrm{M}$ & Metallic FB & M chelonei & Amik & None & $20 / 20$ & - \\
\hline 17 & $55 / \mathrm{F}$ & Pterygium excision & $M$ chelonei & Amik & EK/F & $20 / 60$ & + \\
\hline 18 & $30 / \mathrm{M}$ & Metallic FB & M chelonei & Amik & EK & $20 / 25$ & + \\
\hline 19 & $55 / \mathrm{M}$ & Metallic FB & M chelonei & Amik & EK twice & $20 / 100$ & + \\
\hline 20 & $34 / F$ & Metallic FB & M chelonei & Amik & EK & $20 / 40$ & - \\
\hline 21 & $26 / \mathrm{M}$ & Clay FB & $M$ chelonei & Amik & None & $20 / 25$ & + \\
\hline 22 & $35 / \mathrm{M}$ & Metallic FB & M chelonei & Amik & EK & $20 / 30$ & - \\
\hline
\end{tabular}

FB = foreign body; EK = extirpative keratectomy; F = conjunctival flap; VA = visual acuity; Amik = amikacin; Cipro = ciprofloxacin.

${ }^{\star}$ By direct microscopy, Ziehl-Nielsen stain.

times daily and cefazolin $50 \mathrm{mg} / \mathrm{ml}$ eyedrops every hour around the clock. The keratitis continued to worsen on this regimen. Viral, bacterial, and fungal cultures were negative; ZiehlNielsen stained specimens were also negative. The Löwenstein-Jensen medium grew $M$ fortuitum after 7 days. The patient was started on topical amikacin $50 \mathrm{mg} / \mathrm{ml}$ hourly around the clock, as an addition to the cefazolin eyedrops already in use. Ten days later, the patient underwent anterior lamellar keratectomy in the right eye because of what we perceived to be a poor clinical response (that is, continued stromal inflammation, infiltration, and poor wound healing) of the infection to the medical therapy. Amikacin and cefazolin were continued after the keratectomy. He responded well to the extirpative therapy and his cornea epithelialised completely within 2 weeks, leaving only a faint anterior scar. Histopathological examination and Löwenstein-Jensen cultures of the surgical specimen did not show any residual organisms within the stromal lamellae. The final visual acuity at the latest examination a year later was 20/100 in the right eye.

\section{Case 20}

While riding her motorcycle, a 34-year-old woman sustained a small, superficial, metallic foreign body injury to her left cornea. This was removed that same day by a general medical doctor. Five days later, the patient developed pain, photophobia, and tearing in the injured eye. Her doctor noted a corneal ulcer and referred the woman to us for evaluation and treatment.

On examination, her visual acuity was $20 / 20$ in the right eye and 20/200 in the left. Moderate conjunctival injection of the left eye was noted and slit-lamp biomicroscopy showed a 3 $\times 3 \mathrm{~mm}$ central corneal epithelial defect with a 'cracked windshield' anterior stromal infiltrate (Fig 2).

We suspected non-tuberculous mycobacterial keratitis and immediately started the patient on hourly doses of topical amikacin 50 $\mathrm{mg} / \mathrm{ml}$. Our suspicion was confirmed 1 week later with the growth of $M$ chelonei in the Löwenstein-Jensen medium. The organism was sensitive in vitro to amikacin. Resolution of the infiltrate appeared to come to a standstill after initial improvement the first 5 days. A very superficial lamellar keratectomy (essentially subepithelial) was performed 4 days later in order to debulk the infiltrate and to improve topical drug penetration. The cornea reepithelialised within 2 weeks and the patient was left with good uncorrected visual acuity of $20 / 40$ in the left eye. A faint, mid-stromal scar remained in the cornea.

\section{Discussion}

Non-tuberculous mycobacteria are aerobic, non-spore forming, non-motile bacilli. Owing to the many differences in characteristics from the 'typical' $M$ tuberculosis, non-tuberculous mycobacteria have been called atypical, anonymous, or unclassified. ${ }^{24}$ In the past, most nontuberculous mycobacteria were thought to be environmental saprophytes, widely distributed in soil and water, and incapable of producing clinical infections.

In 1959 , Runyon ${ }^{1}$ proposed a classification of non-tuberculous mycobacteria, under which group IV is the $M$ fortuitum complex, consisting of $M$ fortuitum, $M$ chelonei, $M$ smegmatis, and $M$ vaccae. ${ }^{24}$ Non-tuberculous mycobacteria have been isolated from the normal flora of human sputum, gastric contents, and ocular surfaces. ${ }^{18}$ Both $M$ fortuitum and $M$ chelone $i$ are responsible for a growing number of skin and soft tissue diseases. The rise in the number of reported cases is probably due to: (1) better understanding of the clinical features and enhanced awareness of diseases caused by this organism, (2) better diagnostic methods and criteria, and (3) increased iatrogenic or nosocomial infections associated with surgery, renal dialysis, and immunosuppression. Surgical debridement is now widely advocated for the treatment of postoperative infections and other outbreaks of nosocomial infections..$^{25}$ In the eye, non-tuberculous mycobacterial 
keratitis occurs most often after corneal trauma, especially those involving foreign

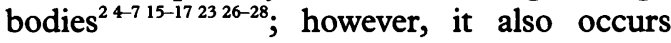
after anterior segment surgery, including radial keratotomy, ${ }^{8}$ penetrating keratoplasty, ${ }^{11819}$ incisional posterior lens capsulotomy, ${ }^{9}$ and in corneal suture abscesses. ${ }^{91029}$ Contact lenses have also been causally associated with nontuberculous mycobacterial keratitis. ${ }^{318} 30$ Several investigators have advocated surgical extirpation therapy in runaway corneal infections that respond poorly to medical therapy. $^{36-9121819272931-33}$

In our present experience with nontuberculous mycobacterial corneal infections, we have learned about the important role of extirpative lamellar keratectomy in the treatment of keratitis recalcitrant to antimicrobial medical therapy alone. This procedure debulks the microbial inoculum, facilitates drug penetration, removes optical opacities, provides tissue for cultures and histopathological examination, and allows the eye to stabilise and become quiescent before it undergoes a more extensive operation such as corneal transplantation. In our experience, it sometimes may even obviate the need for lamellar or penetrating keratoplasty altogether. Our findings parallel those of recent reports. ${ }^{32} 33$

If anterior keratectomy fails, a conjunctival flap appears to be very effective in stopping the infection. The flap could be taken down for restoration of vision in the future, either with or without the use of corneal transplantation. We believe the overlying conjunctiva allows a more direct contiguity of the intrastromal mycobacteria to the cell mediated immune mechanisms.

We were initially surprised by the high incidence $(50 \%)$ of negative acid fast staining with the Ziehl-Nielsen stain. At the University of Michigan, we have begun using the FiteFerraco stain in delineating non-tuberculous mycobacteria. This method uses a gentler acid wash than the Ziehl-Nielsen technique, thus allowing the bacilli to better retain their stain..$^{34}$ For future cases of non-tuberculous mycobacterial keratitis, we plan to rely predominantly on the Fite-Ferraco stain and LöwensteinJensen cultures.

Both $M$ fortuitum and $M$ chelone $i$ are resistant to most antibiotics, including the standard antituberculous drugs (for example, isoniazid, streptomycin, rifampin, and ethambutol). For more than 20 years, amikacin has been the single most frequently used topical antimicrobial drug in the treatment of non-tuberculous mycobacterial keratitis. ${ }^{49101418-21}$ Dalovisio and associates ${ }^{4}$ reported highly successful treatment of infections with amikacin, especially when used in conjunction with a second antibiotic, such as doxycycline. Thus, it may be advantageous to treat the keratitis with topical amikacin and add a second agent, such as kanamycin, tetracycline or its relatives, fluoroquinolones, erythromycin, vancomycin, cephalosporins, sulphonamides, neomycin, or clarithromycin. ${ }^{9182022}$ The benefits of two drug synergy may not be obvious on routine in vitro testing.
Keratitis caused by non-tuberculous mycobacteria often manifests a relatively indolent, recalcitrant stromal infection which develops with a 2 to 3 week delay after trauma or surgery. The disease may wax and wane over a period of several months. ${ }^{6328}$ Corticosteroids are likely to aggravate the infectious process. Pain is variable, but is certainly never excruciating as in Acanthamoeba keratitis.

The corneal stromal infiltrates may show feathered edges, satellite lesions, crystalline keratopathy, or a ring pattern. Satellite lesions and feathery edges of infiltrates may cause confusion with a fungal process. The crystalline keratopathic changes are due to colonisation of the organisms along discrete lamellar planes in the stroma-that is, the organisms spread along the paths of least resistance. ${ }^{35}$ This peculiar intrastromal distribution is thought to be associated with a combination of low virulence of the involved micro-organism and suppression of the 'white cell response' by corticosteroids. ${ }^{35}$ Three of our patients manifested crystalline keratopathy. Two such patients had a cracked windshield infiltrative pattern, which in actuality is a main infiltrate surrounded by a radiating pattern of crystalline keratopathy. The cracked windshield pattern is thought by some to be diagnostic of nontuberculous mycobacterial keratitis. ${ }^{123}$ Partial or complete ring infiltrates are common with this infection and may cause confusion with herpetic, fungal, or Acanthamoeba keratitis. It is unclear whether this ring is the result of an immune deposition or due to other causes. The corneal epithelium has been reported to be intact over stromal infiltrates in a number of cases reported elsewhere ${ }^{6}$; however, none of our patients had intact epithelium.

Iritis with or without hypopyon was commonly observed in our patients. The degree of anterior chamber inflammation appears to parallel the gravity of the events in the cornea, and is probably a reactive phenomenon rather than infectious per se.

We did not encounter any cases of infectious scleritis in our series. The only reported case of non-tuberculous mycobacterial scleritis in the literature is a sclerokeratitis occurring contiguous to a limbal corneal infection. ${ }^{17}$ None of the corneal infiltrates in our patients were peripheral enough to straddle the limbus.

In summary, non-tuberculous mycobacterial keratitis should be suspected in corneal ulcers developing after foreign body injuries (especially metallic ones), particularly if the ulcer is indolent and fails to respond adequately to common antibacterial, antifungal, antiviral, and antiparasitic drugs. Surgically acquired non-tuberculous mycobacterial infections, although less common, have the potential to occur in epidemic clusters in surgical facilities. $^{89}$ Better awareness of the disease and improved familiarity with diagnostic and therapeutic methods are important factors in reducing the incidence of debilitating ocular damage from this infection. Misdiagnoses are common and may lead to crucial delays in therapy. The Fite-Ferraco stain should perhaps be used instead of the Ziehl-Nielsen stain. 
Löwenstein-Jensen cultures should not be discarded after only 1 week, but should be retained for 4 weeks if necessary to allow colonies adequate time to grow. Although $M$ chelonei and fortuitum are rapid growers on subculture, the initial isolation can be slow as seen in the three cases $(5,7$, and 20$)$ detailed in the Results section. Despite excellent in vitro activity of amikacin against non-tuberculous mycobacteria, the clinical response is often unpredictable ${ }^{32}$ and does not correlate well with the minimum inhibitory concentration (MIC). $\mathrm{Hu}^{32}$ postulated that poor drug penetration into the cornea, emergence of resistant strains, immune ulceration, and stromal necrosis impeding epithelialisation and wound healing may all contribute to this discrepancy. In refractory cases showing no visible improvement after 1 week of conservative therapy, surgical extirpative therapy should be strongly considered. Conjunctival flap surgery appears efficacious if extirpative surgery fails to control the infection. Penetrating corneal transplantation $\dot{a}$ chaud may be an additional mode of therapy for the very deep infections close to Descemet's membrane; however, if possible, it is best to delay transplantation until the infection is eradicated and the eye is quiescent.

The authors have no proprietary interest in any of the drugs or materials used in this study.

1 Runyon EH. Anonymous mycobacteria in pulmonary disease. Med Clin North Am 1959;43:273-8.

2 Turner L, Stinson I. Mycobacterium fortuitum as a cause of corneal ulcer. Am $\mathcal{f}$ Ophthalmol 1965;60:329-31.

3 Gangadharam PRJ, Lanier JD, Jones DB. Keratitis due to Gangadharam PRJ, Lanier JD, Jones DB. Keratiti

4 Dalovisio JR, Pankey GA, Wallace RJ Jr, Jones DB. Clinical usefulness of amikacin and doxycycline in the treatment of infection due to Mycobacterium fortuitum and Mycobacterium chelonei. Rev Infect Dis 1981;3:1068-74.

5 Wallace RJ Jr, Jones DB, Wiss $\mathrm{K}$. Sulfonamide activity against Mycobacterium fortuitum and Mycobacterium chelonei. Rev Infect Dis 1981;3:898-904.

6 Turner L. Atypical mycobacteria infections in ophthalmology. Trans Am Ophthalmol Soc 1970;68:667-729.

7 Meisler DM, Friedlaender MH, Okumoto M. Mycobacterium chelonei keratitis. Am f Ophthalmol 1982;94:398-401.

8 Robin JB, Beatty RF, Dunn S, Trousdale MD, Riffenburgh R, Rao N, et al. Mycobacterium chelonei keratitis after radial keratotomy. Am f Ophthalmol 1986;102:72-9.

9 Newman PE, Goodman RA, Waring GO III, Finton RJ, Wilson LA, Wright J. A cluster of cases of Mycobacterium chelonei keratitis associated with outpatient office procedures. Am $₹$ Ophthalmol 1984;97:344-8.

10 Laflamme MY, Poisson M, Chehade N. Mycobacterium chelonei keratitis following penetrating keratoplasty. Can $\mathcal{F}$ Ophthalmol 1987;22:178-80.
11 Aylward GW, Stacey AR, Marsh RJ. Mycobacterium chelonei infection of a corneal graft. Brf Ophthalmol 1987 71:690-3.

12 Mirate DJ, Hull DS, Steel JH Jr, Carter MJ. Mycobacterium chelonei keratitis: a case report. $\mathrm{Br} \mathcal{F}$ Ophthalmol 1983;67: 324-6.

13 Zabel RW, Mintsioulis G, MacDonald I. Mycobacterium chelonei keratitis in a soft-contact lens wearer. Can $7 \mathrm{Oph}$ chelonei keratitis in a sof

14 Bullington RH Jr, Lanier JD, Font RL. Non-tuberculous mycobacterial keratitis. Arch Ophthalmol 1992;110:519-24.

5 Knapp A, Stern GA, Hood C I. Mycobacterium aviumintracellulare corneal ulcer. Cornea 1987;6:175-80.

16 Moore MB, Newton C, Kaufman HE. Chronic keratitis caused by Mycobacterium gordonae. Am f Ophthalmol 1986;102:516-21.

17 Schonherr U, Naumann GO, Lang GK, Bialasiewicz AA Sclerokeratitis associated with Mycobacterium marinum. Am $\mathcal{F}$ Ophthalmol 1989;108:607-8.

18 Dugel PU, Holland GN, Brown HH, Pettit TH, Hofbaue JD, Simons KB, et al. Mycobacterium fortuitum keratitis. Am $\mathcal{f}$ Ophthalmol 1988;105:661-9.

19 Matoba A. Mycobacterium chelonei keratitis. Am $f$ Ophthalmol 1987;103:595-6.

20 Helm CJ, Holland GN, Lin R, Berlin OG, Bruckner DA Comparison of topical antibiotics for treating Mycobacterium fortuitum keratitis in an animal model. Am $\mathcal{f} O p h t h a l-$ mol 1993;116:700-7.

21 Stevens RK, Holland GN, Paschal JF, Phinney RB, Berlin OG, Bruckner DA. Mycobacterium fortuitum keratitis. A comparison of topical ciprofloxacin and amikacin in an animal model. Cornea 1992;11:500-7.

22 Lin R, Holland GN, Helm CJ, Elias SJ, Berlin OG, Bruckner DA, et al. Comparative efficacy of topical ciprofloxacin for treating Mycobacterium fortuitum and Mycobacterium chelonei keratitis in an animal model. Am $\mathcal{f}$ Ophthalmo 1994;117:657-62.

23 Lazar M, Nemet P, Bracha R, Campus A. Mycobacterium fortuitum keratitis. Am $\mathcal{F}$ Ophthalmol 1974;78:530-2.

24 Sommers HM, Good RC. Mycobacterium. In: Lennette EH, Balows A, Hausler WJ Jr, eds. Manual of clinical microbiology, 4th ed. Washington, DC: American Society of biology, 4th ed. Washingto
Microbiology, 1985;216-48.

25 Wallace RJ Jr, Swenson JM, Silcox VA, Good RC, Tschen JA, Stone MS. Spectrum of disease due to rapidly growing mycobacteria. Rev Infect Dis 1983;5:657-9.

26 Lauring LM, Wergeland FL, Sack GE. Anonymous mycobacterium keratitis. Am f Ophthalmol 1969;67:130-3

27 Levenson DS, Harrison CH. Mycobacterium fortuitum corneal ulcer. Arch Ophthalmol 1966;75:189-91.

28 Willis WE, Laibson PR. Intractable Mycobacterium fortuitum corneal ulcer in man. Am $\mathcal{F}$ Ophthalmol 1971;71:5004.

29 Wunch SE, Boyle GL, Leopold IH, Littman ML. Mycobacterium fortuitum infection of corneal graft. Arch Ophthalterium fortuitum in

30 Kent HD, Cohen EJ, Laibson PR, Arentsen JJ. Microbial keratitis and corneal ulceration associated with therapeutic soft contact lenses. $C L A O \mathcal{F} 1990 ; 16: 49-52$.

$31 \mathrm{Hu}$ FR. Infectious crystalline keratopathy caused by Mycobacterium fortuitum and Pseudomonas aeruginosa. Am Ophthalmol 1990;109:738-9.

$32 \mathrm{Hu}$ FR. Extensive lamellar keratectomy for treatment of nontuberculous mycobacterial keratitis. Am $\mathcal{F}$ Ophthalmol 1995;120:47-54.

33 Tseng SH, Hsiao WC. Therapeutic lamellar keratectomy in the management of nontuberculous mycobacterium keratitis refractory to medical treatment. Cornea 1995;14:161-6.

34 Fite GL, Honolulu TH. The fuchsin-formaldehyde method of staining acid-fast bacilli in paraffin sections. $f \mathrm{Lab} \mathrm{Clin}$ Med 1940;25:743-4.

35 Gorovoy MS, Stern GA, Hood CI, Allen C. Intrastromal noninflammatory bacteria colonization of a corneal graft. Arch Ophthalmol 1983;101:1749-52. 